\title{
Olanzapine in the treatment of hallucinosis in idiopathic parkinson's disease: a cautionary note
}

\author{
Jacqueline M Graham, Jon D Sussman, Kay S Ford, Harvey J Sagar
}

\begin{abstract}
Background-Hallucinosis is a dopaminergic dose limiting complication of the treatment of idiopathic Parkinson's disease. Typical neuroleptic medications cannot be used for suppressing hallucinosis because the extrapyramidal side effects worsen parkinsonian motor control. Olanzapine is a novel atypical antipsychotic drug with few reported extrapyramidal side effects which may be more suitable for controlling hallucinosis in these patients.
\end{abstract}

Methods-Olanzapine was given to five patients with idiopathic Parkinson's disease and the dosage was titrated until a clinically meaningful reduction in hallucinosis was achieved. The commercially available $5 \mathrm{mg}, 7.5 \mathrm{mg}$ and $10 \mathrm{mg}$ tablets were used.

Results-After an initial 9 days of treatment, hallucinosis frequency was significantly reduced, an effect which was maintained with continued treatment. However, during this early phase of treatment, parkinsonian motor disability increased, which resulted in two of the patients discontinuing medication.

Conclusions-Olanzapine is effective in the suppression of hallucinosis in patients with idiopathic Parkinson's disease but the currently available dose increments may result in an unacceptable exacerbation of motor disability.

(F Neurol Neurosurg Psychiatry 1998;65:774-777)

Keywords: Parkinson's disease; hallucinosis; olanzapine

Neurology, N Floor,

oyal Hallamshire

Road, Sheffield, UK

J M Graham

J D Sussman

K S Ford

H J Sagar

Correspondence to:

Professor Harvey J Sagar,

University of Sheffield,

Department of Clinical

Neurology, N Floor, Royal

Hallamshire Hospital,

Glossop Road, Sheffield

S10 2JF, UK.

Received 27 October 1997 and in revised form

7 April 1998

Accepted 16 April 1998

movement primarily resulting from dys tion of the dopaminergic nigrostriatal system. Dopaminergic therapy ameliorates the motor dysfunction of Parkinson's disease but may induce hallucinosis by overstimulating the dopaminergic mesocorticolimbic system. ${ }^{1}$ Typical neuroleptic drugs which antagonise dopamine in both the nigrostriatal and mesocorticolimbic systems provide effective relief from psychosis but carry the risk of extrapy- ramidal side effects which complicate their use in Parkinson's disease. ${ }^{2}$ Olanzapine has an atypical profile, binding to dopaminergic $\mathrm{D}_{4}$ and serotonergic $5-\mathrm{HT}_{2}$ receptors in preference to dopaminergic $\mathrm{D}_{2}$ receptors. This results in selective mesolimbic dopamine receptor blockade and minimises extrapyramidal side effects. ${ }^{3}$ The only study to examine olanzapine in the treatment of psychosis in Parkinson's disease suggested that it is well tolerated and causes no deterioration in parkinsonian motor disability. ${ }^{4}$ This study however, benefited from the flexibility of $1 \mathrm{mg}$ olanzapine tablets, which are not commercially available. The aim of our study was to investigate the practicality, efficacy, and adverse events of the commercially available 5 $\mathrm{mg}, 7.5 \mathrm{mg}$, and $10 \mathrm{mg}$ olanzapine tablets on hallucinosis and motor dysfunction in patients with Parkinson's disease.

\section{Methods}

PATIENTS

Five outpatients with idiopathic Parkinson's disease, diagnosed according to the Parkinson's Disease Society Brain Research Centre diagnostic clinical criteria, ${ }^{5}$ and with clinically significant hallucinosis were included in the study. All patients gave informed consent to participate and the study was approved by the South Sheffield research ethics committee.

\section{MEASURES}

Psychosis measures

The psychosis diary (appendix), designed by the authors, monitored the frequency and severity of vivid dreams, hallucinosis (comprising true hallucinations and illusions), and delusions throughout the study. Each event was classified according to whether the content was recognisable, and according to whether fear was invoked. The time of day that each event occurred was also recorded.

\section{Motor measures}

The unified Parkinson's disease rating scale (UPDRS) motor subscale ${ }^{6}$ measured the severity of parkinsonian motor symptoms. The alternate finger tapping test assessed finger dexterity by recording the number of alternate taps on two keys in a 30 second period. The 
Hoehn and Yahr staging ${ }^{7}$ identified the stage of progression of Parkinson's disease. All motor assessments were conducted by a trained observer when the patient was in the "on" state.

Demographics

Details of age at onset of disease, duration of disease, dopaminergic therapy, and concurrent medical complaints were recorded.

Clinical measures

Blood pressure, haematology, biochemistry, and adverse events were monitored throughout the study.

PROCEDURE

Olanzapine was initiated at a daily dose of $5 \mathrm{mg}$ and titrated fortnightly in $2.5 \mathrm{mg}$ increments to optimisation at day 65 . The optimised daily dose of olanzapine was reached when either hallucinosis was suppressed completely or when a reduction in hallucinosis frequency and severity acceptable to the patient was achieved. From day 65 to day 111 dopaminergic therapy was increased, if required, to improve motor control.

\section{Results}

CASE 1

A 74 year old man with Hoehn and Yahr stage 3 Parkinson's disease of 10 years duration was experiencing non-threatening visual hallucinosis twice a day and frightening vivid dreams while receiving $500 \mathrm{mg}$ levodopa and $3 \mathrm{mg}$ pergolide daily. Olanzapine at $5 \mathrm{mg}$ daily for 9 days reduced hallucinosis frequency to about once a day and completely prevented vivid dreaming. Motor disability had, however, increased from a UPDRS score of 25 to 49 . Olanzapine was not titrated beyond $5 \mathrm{mg}$ and, at day 20 , poor mobility and double incontinence necessitated his withdrawal from the study. Hallucinosis recurred on olanzapine withdrawal, accompanied by episodes of fluctuating confusion and persisting incontinence.

CASE 2

A 73 year old woman with Hoehn and Yahr stage 4 Parkinson's disease of 29 years duration was experiencing frightening visual hallucinations up to four times a day while medicated with $900 \mathrm{mg}$ levodopa and $5 \mathrm{mg}$ pergolide daily. After 9 days of olanzapine at the initial daily dose of $5 \mathrm{mg}$, hallucinosis was occurring less than once a day but parkinsonian motor symptoms had increased in severity from a UPDRS score of 27 to 67 and time spent in the "off" state had increased from $25 \%$ to over $75 \%$ of the waking day. With her agreement, olanzapine was continued at $5 \mathrm{mg}$ a day. On day 30 she had an episode of loss of consciousness. Blood pressure at day 33 was $106 / 60 \mathrm{~mm}$ $\mathrm{Hg}$ supine and $84 / 77 \mathrm{~mm} \mathrm{Hg}$ standing, by contrast with $128 / 64 \mathrm{~mm} \mathrm{Hg}$ supine and $120 / 70 \mathrm{~mm} \mathrm{Hg}$ standing at study entry.
Olanzapine was discontinued on day 35. The pretreatment mobility state returned but with no recurrence of hallucinations.

CASE 3

A 66 year old man with Hoehn and Yahr stage 4 Parkinson's disease of 29 years duration was experiencing florid visual, auditory, and tactile hallucinosis for up to 4 hours each day while receiving $600 \mathrm{mg}$ levodopa, $5 \mathrm{mg}$ pergolide, and $90 \mathrm{mg}$ subcutaneous apomorphine daily. Olanzapine was titrated up to $7.5 \mathrm{mg}$ daily by day 65, whereupon transient hallucinations of reduced severity were occurring less than twice a day. Motor disability was, however, increased from an initial UPDRS score of 49 to a score of 70 by day 65 . Apomorphine titration up to 100 mg improved motor control, although not to

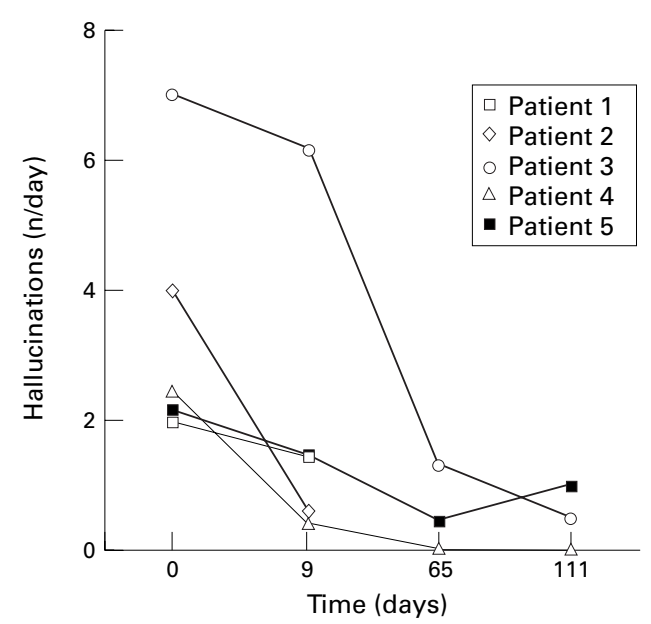

Figure 1 Daily frequency of hallucinosis at study entry, after 9 days of olanzapine at $5 \mathrm{mg}$ a day, after olanzapine titration to day 65, and after an increase in dopaminergic treatment to day 111.

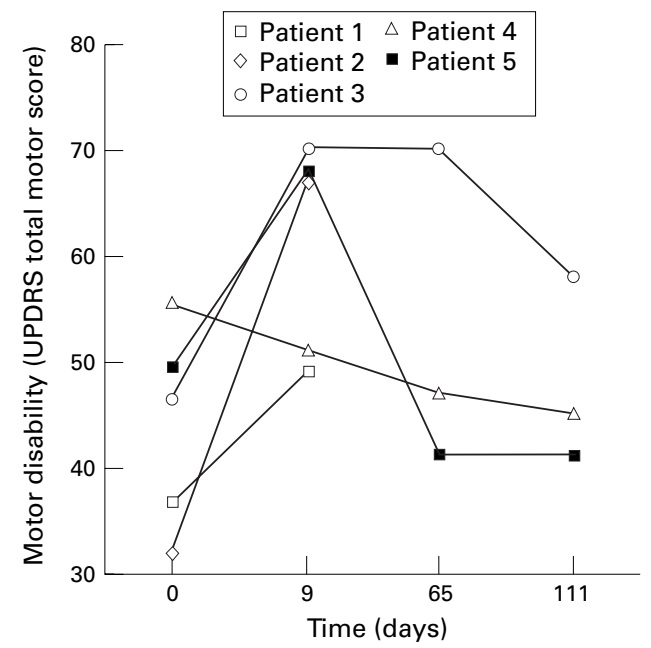

Figure 2 Parkinsonian motor disability, as measured by the UPDRS total motor score, at study entry, after 9 days of olanzapine at $5 \mathrm{mg}$ a day, after olanzapine titration to day 65, and after an increase in dopaminergic treatment to day 111. 
the prestudy level, without increasing the frequency or severity of hallucinosis.

CASE 4

A 70 year old woman with Hoehn and Yahr stage 5 Parkinson's disease of 9 years duration was experiencing frightening visual and tactile hallucinosis up to three times a day while receiving $800 \mathrm{mg}$ levodopa daily. The optimised daily dose of olanzapine at day 65 was 5 mg. Hallucinosis had ceased completely by day 14 and, although speech became quieter throughout the period of olanzapine titration, her undertreated parkinsonian motor symptoms were otherwise unaffected. Levodopa was titrated to $1200 \mathrm{mg}$ daily without recurrence of hallucinosis, but with no benefit to mobility.

CASE 5

A 53 year old woman with Hoehn and Yahr stage 5 Parkinson's disease of 18 years duration was experiencing threatening visual and auditory hallucinations and vivid dreams about twice a day while receiving $200 \mathrm{mg}$ levodopa and $82 \mathrm{mg}$ subcutaneous apomorphine daily. Olanzapine was titrated to $7.5 \mathrm{mg}$ daily by day 65 . Vivid dreams were suppressed by day 15 and only hallucinosis of reduced severity, occurring less than once a day, persisted at day 65. Olanzapine, at $5 \mathrm{mg}$ daily, over days 1 to 9 led to an increase in UPDRS motor score from 50 to 68 , but this returned to the pretreatment level by day 65 . The addition of $100 \mathrm{mg}$ levodopa improved finger dexterity without increasing the frequency or severity of hallucinosis.

Figure 1 illustrates the daily frequency of hallucinosis at study entry, after 9 days of olanzapine at $5 \mathrm{mg}$ daily, after olanzapine titration to day 65, and after an increase in dopaminergic treatment to day 111. The frequency of hallucinosis was significantly reduced after 9 days treatment with olanzapine at $5 \mathrm{mg}$ daily (Wilcoxon $\mathrm{T}=0, \mathrm{p}<0.032$ ). This reduction was maintained at both day 65 and day 111

Figure 2 illustrates parkinsonian motor disability at study entry, after 9 days of olanzapine at $5 \mathrm{mg}$ daily, after olanzapine titration to day 65 , and after an increase in dopaminergic treatment to day 111. There was a trend for motor disability to increase after 9 days of treatment with 5 $\mathrm{mg}$ of olanzapine daily (Wilcoxon $\mathrm{T}=1$, $\mathrm{p}<0.063)$. However, it is unclear whether this increase persisted at day 65 or if it was improved after an increase in dopaminergic treatment to day 111.

\section{Discussion}

This study confirms the utility of olanzapine for the suppression of hallucinosis in patients with Parkinson's disease. However, by contrast with the findings of Wolters et $a l^{4}{ }^{4}$ olanzapine produced an increase in parkinsonian motor disability when treatment was initiated at a dose of $5 \mathrm{mg}$ daily. We propose that the increase in parkinsonian motor disability is due to the large initial olanzapine dose of 5 mg. The $\mathrm{D}_{2}$ receptor affinity of olanzapine, although lower than that of traditional neuroleptic preparations, may be high enough to antagonise the nigrostriatal receptors to a degree which increases motor disability when initiated at $5 \mathrm{mg}$ daily, but not when titrated from a lower starting dose. With the lower initial dose and smaller incremental titration of olanzapine, the nigrostriatal $\mathrm{D}_{2}$ receptors may be capable of increasing sensitivity by upregulation.

An increase in dopaminergic therapy can be tolerated during olanzapine treatment, without intensifying hallucinosis. This finding is in agreement with that of Wolters $e t a l^{4}$ and shows an important benefit of olanzapine as hallucinosis is often a dose limiting complication of dopaminergic therapy in Parkinson's disease.

In conclusion, olanzapine seems effective in suppressing hallucinosis in patients with idiopathic Parkinson's disease. It has the additional benefit of allowing an increase in dopaminergic therapy without the exacerbation of hallucinosis. However $5 \mathrm{mg}$, which is the lowest commercially available dose of olanzapine, can increase motor disability and may limit the suitability of this treatment for patients with idiopathic Parkinson's disease. Further studies are required to determine whether an increase in dopaminergic dose at the initiation of olanzapine therapy offsets the motor deterioration without compromising the benefit to hallucinosis.

1 Moskovitz C, Moses H, Klawans HL. Levodopa-induced psychosis: A kindling phenomenon. Am $\mathcal{f}$ Psychiatry 1978;135:669-75.

2 Creese I, Burt DR, Snyder SH. Dopamine receptor binding predicts clinical and pharmacological potencies of antischizophrenic drugs. Science 1978;192: 481-3.

3 Beasley CM Jr, Tollefson G, Tran P, et al. Olanzapine versus placebo and haloperidol. Acute phase results of the North American double-blind olanzapine trial. Neuropsychopharmacology 1996;14:111-24.

4 Wolters ECh, Jansen ENH, Tuynman-Qua H, et al. Olanzapine in the treatment of dopaminomimetic psychosis in patients with Parkinson's disease. Neurology 1996;47:1085-

5 Daniel SE, Lees AJ. Parkinson's Disease Society brain bank, London: overview and research. F Neural Transm 1993; 39(suppl):165-72.

6 Fahn S, Elton RL, members of the UPDRS Development Committee. The unified Parkinson's disease rating scale. In: Fahn S, Marsden CD, Calne DB, et al, eds. Recent developments in Parkinson's disease. Vol 2. Florham Park, NJ: Macmillan Healthcare Information, 1987:153-163, 293304.

7 Hoehn MM, Yahr MD. Parkinsonism: onset, progression, and mortality. Neurology 1967;17:427-42. 


\section{Appendix: the psychosis diary}

Definition of terms

Vivid dream $=$ when asleep, a dream with unusually vivid content which may or may not be frightening.

Hallucination $=$ a perception which is only available to you. For example, a figure only you can see, or an object you perceive in a different form.

Delusion = an idea in which you strongly believe without strong evidence and which your family insists is false.

Directions

Decide which number $(1,2,3,4)$ best describes the vivid dream, hallucination, or delusion and enter it in the row showing the time of day it occurred. If more than one experience occurs in a time slot, record each event separately.

\section{Vivid dreams}

$1=$ unknown theme, not frightening $2=$ known theme, not frightening

$3=$ unknown theme, frightening

$4=$ known theme, frightening

\section{Hallucinations}

$1=$ unrecognisable, not frightening

$2=$ well formed, not frightening

$3=$ unrecognisable, frightening

$4=$ well formed, frightening

\section{Delusions}

$1=$ not threatening, unconcerning

$2=$ threatening, unconcerning

$3=$ threatening, concerning

\begin{tabular}{|c|c|c|c|c|}
\hline Day & Time & Vivid dream & Hallucination & Delusion \\
\hline \multirow[t]{8}{*}{1} & $12 \mathrm{AM}-3 \mathrm{AM}$ & & & \\
\hline & $3 \mathrm{AM}-6 \mathrm{AM}$ & & & \\
\hline & $6 A M-9 A M$ & & & \\
\hline & $9 \mathrm{AM}-12 \mathrm{PM}$ & & & \\
\hline & $12 \mathrm{PM}-3 \mathrm{PM}$ & & & \\
\hline & $3 P M-6 P M$ & & & \\
\hline & $6 P M-9 P M$ & & & \\
\hline & $9 \mathrm{PM}-12 \mathrm{AM}$ & & & \\
\hline \multirow[t]{8}{*}{2} & $12 \mathrm{AM}-3 \mathrm{AM}$ & & & \\
\hline & $3 A M-6 A M$ & & & \\
\hline & $6 \mathrm{AM}-9 \mathrm{AM}$ & & & \\
\hline & $9 \mathrm{AM}-12 \mathrm{PM}$ & & & \\
\hline & $12 \mathrm{PM}-3 \mathrm{PM}$ & & & \\
\hline & $3 P M-6 P M$ & & & \\
\hline & $6 P M-9 P M$ & & & \\
\hline & $9 \mathrm{PM}-12 \mathrm{AM}$ & & & \\
\hline
\end{tabular}

